\title{
Superioridade da PET/CT com FNa-F18 na Deteção de Metástases Ósseas quando Comparada com Outros Métodos de Diagnóstico por Imagem
}

\section{Superiority of $18 \mathrm{~F}-\mathrm{FNa} \mathrm{PET} / \mathrm{CT}$ for Detecting Bone Metastases in Comparison with Other Diagnostic Imaging Modalities}

\author{
Paula LAPA $\triangle^{1}$, Tiago SARAIVA ${ }^{1}$, Rodolfo SILVA ${ }^{1,4}$, Margarida MARQUES ${ }^{2,3}$, Gracinda COSTA ${ }^{1}$, João Pedroso LIMA ${ }^{1,4}$ \\ Acta Med Port 2017 Jan;30(1):53-60 - http://dx.doi.org/10.20344/amp.7818
}

\section{RESUMO}

Introdução: A tomografia por emissão de positrões/tomografia computorizada - FNa-F18 vem sendo considerada como uma modalidade imagiológica com vantagens na pesquisa de metastização óssea. Comparámos a sua capacidade para deteção de metástases ósseas com a de outras técnicas imagiológicas.

Material e Métodos: Avaliámos retrospetivamente 114 doentes que realizaram tomografia por emissão de positrões/tomografia computorizada - FNa-F18. Destes, 49 realizaram também cintigrafia óssea, 61 tomografia por emissão de positrões/tomografia computorizada - FDG-F18 e 10 tomografia por emissão de positrões/tomografia computorizada - FCH-F18. Identificámos a técnica que detetou um maior número de metástases ósseas. Comparámos ainda a tomografia por emissão de positrões com a componente tomografia computorizada da tomografia por emissão de positrões/tomografia computorizada - FNa-F18. Registámos as situações nas quais a tomografia por emissão de positrões/tomografia computorizada FNa-F18 e a cintigrafia óssea necessitaram de exames adicionais para esclarecimento complementar.

Resultados: A tomografia por emissão de positrões/tomografia computorizada - FNa-F18 foi superior à cintigrafia óssea em $49 \%$ dos doentes $(p<0,001)$; foi superior à tomografia por emissão de positrões/tomografia computorizada - FDG-F18 em $59 \%$ dos doentes $(p<0,001)$ e foi superior à tomografia por emissão de positrões/tomografia computorizada - $\mathrm{FCH}-\mathrm{F} 18$ em $40 \%$ dos doentes $(p<0,001)$. Nenhuma das técnicas imagiológicas avaliadas Ihe foi superior. Na tomografia por emissão de positrões/tomografia computorizada FNa-F18 a componente tomografia por emissão de positrões foi superior à tomografia computorizada em $35 \%$ dos casos $(p<0,001)$. Foi sugerida investigação complementar em apenas 3,5\% dos doentes que realizaram tomografia por emissão de positrões/tomografia computorizada - FNa-F18 (45\% para a cintigrafia óssea) $(p<0,001)$.

Discussão: Em conformidade com o referido por outros autores, a nossa experiência confirma que a tomografia por emissão de positrões/tomografia computorizada - FNa-F18 tem excelente desempenho na deteção de metástases ósseas, sendo capaz de identificar lesões em mais doentes, e em maior número, quando comparada com outras técnicas imagiológicas.

Conclusão: A tomografia por emissão de positrões/tomografia computorizada - FNa-F18 revelou superioridade na deteção de metástases ósseas comparativamente à cintigrafia óssea, à tomografia por emissão de positrões/tomografia computorizada - FDG-F18 e à tomografia por emissão de positrões/tomografia computorizada - FCH-F18.

Palavras-chave: Cintigrafia; Neoplasias Ósseas/diagnóstico por imagem; Neoplasias Ósseas/secundário; Tomografia por Emissão de Positrões

\section{ABSTRACT}

Introduction: The 18F-NaF positron emission tomography/computed tomography is being considered as an excellent imaging modality for bone metastases detection. This ability was compared with other imaging techniques.

Material and Methods: We retrospectively evaluated 114 patients who underwent $18 \mathrm{~F}-\mathrm{NaF}$ positron emission tomography/computed tomography. Of these, 49 patients also had bone scintigraphy, 61 18F-FDG positron emission tomography/computed tomography and $1018 \mathrm{~F}-\mathrm{FCH}$ positron emission tomography/computed tomography. We identified the technique that detected the largest number of bone metastases. For the detection of skeletal metastases with the $18 \mathrm{~F}-\mathrm{NaF}$ positron emission tomography/computed tomography study, the contribution of the positron emission tomography component was compared with the contribution of the computed tomography component. Cases in which 18F-NaF positron emission tomography/computed tomography and bone scintigraphy required further additional tests for diagnosis clarification were registered.

Results: The 18F-NaF positron emission tomography/computed tomography was superior to bone scintigraphy in $49 \%$ of the patients $(p<0.001)$; it was superior to 18 F-FDG positron emission tomography/computed tomography in $59 \%$ of the patients $(p<0.001)$ and it was superior to $18 \mathrm{~F}-\mathrm{FCH}$ positron emission tomography/computed tomography in $40 \%$ of the patients $(p<0.001)$. None of the compared imaging techniques were superior to $18 \mathrm{~F}-\mathrm{NaF}$ positron emission tomography/computed tomography. The positron emission tomography component was superior to computed tomography in $35 \%$ of the cases $(p<0.001)$. Further investigation was suggested in only $3.5 \%$ of patients who underwent $18 \mathrm{~F}-\mathrm{NaF}$ positron emission tomography/computed tomography ( $45 \%$ for bone scintigraphy) $(p<0.001)$.

Discussion: As with other authors, our experience also confirms that $18 \mathrm{~F}-\mathrm{NaF}$ positron emission tomography/computed tomography

1. Serviço de Medicina Nuclear. Centro Hospitalar e Universitário de Coimbra. Coimbra. Portugal.

2. Serviço de Tecnologias e Sistemas Informáticos. Centro Hospitalar e Universitário de Coimbra. Coimbra. Portugal.

3. Laboratório de Bioestatística e Informática Médica. Faculdade de Medicina. Universidade de Coimbra. Coimbra. Portugal.

4. Instituto de Ciências Nucleares Aplicadas à Saúde. Universidade de Coimbra. Coimbra. Portugal.

$\square$ Autor correspondente: Paula Lapa. paula.alexandra.lapa@gmail.com

Recebido: 03 de maio de 2016 - Aceite: 13 de setembro de 2016 | Copyright @ Ordem dos Médicos 2017 
is an excellent imaging modality for the detection of bone metastases, detecting lesions in more patients and more lesions per patient. Conclusion: The 18F-NaF positron emission tomography/computed tomography showed a superior ability for the detection of bone metastases when compared with bone scintigraphy, 18F-FDG positron emission tomography/computed tomography and $18 \mathrm{~F}-\mathrm{FCH}$ positron emission tomography/computed tomography.

Keywords: Bone Neoplasms/diagnostic imaging; Bone Neoplasms/secondary Fluorodeoxyglucose F18; Positron-Emission Tomography; Radionuclide Imaging

\section{INTRODUÇÃO}

A metastização para o esqueleto ocorre em 30\% a 70\% dos doentes com neoplasias malignas, sendo o cancro da mama o principal responsável nas mulheres, o cancro da próstata nos homens, seguindo-se, para ambos, o cancro do pulmão..$^{1,2}$ As metástases ósseas podem ser líticas, exibindo comportamento agressivo e crescimento rápido, ou blásticas, mais indolentes, tendo, cada tumor, tendência para um determinado tipo de lesões. O seu diagnóstico precoce é fundamental porque são causa de importantes sintomas e complicações e a sua presença tem enorme impacto no estadiamento, na abordagem terapêutica, no prognóstico e na qualidade de vida dos doentes. ${ }^{1}$

As modalidades imagiológicas com capacidade para detetar metástases ósseas são a radiografia convencional $(R X)$, a tomografia computorizada (CT), a ressonância magnética (RM), a cintigrafia óssea com difosfonatos marcados com Tecnécio-99m (CO) e a tomografia por emissão de positrões/tomografia computorizada (PET/CT). ${ }^{2,3} \mathrm{~A} R X$ não é recomendada, desde há muito, devido à sua assumida baixa sensibilidade. Para que uma metástase óssea seja detetável em RX é necessária uma alteração considerável da densidade óssea, estimada entre $30 \%$ a $75 \%$. A $\mathrm{CT}$ apresenta sensibilidade superior à $\mathrm{RX}$ mas mantém-se a necessidade da lesão ter que condicionar uma alteração significativa da densidade óssea para permitir a sua deteção. Não é, por isso, eficaz para a deteção precoce das metástases ósseas e é, também, considerada pouco sensível para a identificação de metástases intra-medulares. A RM tem a capacidade de detetar precocemente lesões intra-medulares, mas apresenta sensibilidade limitada, inferior à CT, para a deteção das metástases corticais. ${ }^{3} \mathrm{~A} C \mathrm{CO}$ tem sido a técnica imagiológica indicada como o método a utilizar para a pesquisa de metástases ósseas, estimando-se que seja capaz de as identificar cerca de 2 a 18 meses antes da CT. Contudo, a captação do radiofármaco utilizado em CO ocorre nos locais de aumento da atividade osteoblástica, identificando as metástases dessa natureza, sendo as metástases de natureza lítica uma causa importante de falsos negativos. Por outro lado, condições benignas que cursem com aumento do turnover ósseo tais como fraturas, ou alterações ósseas degenerativas e inflamatórias, são uma importante causa de falsos positivos. ${ }^{2}$ Para a deteção de metástases osteoblásticas, a CO tem sensibilidade elevada no esqueleto periférico mas inferior na coluna vertebral e na bacia e tem uma especificidade assumidamente baixa. ${ }^{4} \mathrm{~A}$ introdução da tomografia por emissão de fotão único (SPET) e, principalmente, da SPET/CT, aumentaram a sensibilidade e a especificidade para valores ligeiramente superiores a 90\%. No entanto, a SPET/CT, ao contrário da PET/CT, não é um estudo de corpo inteiro, conseguindo-se aqueles valores, mais favoráveis, apenas na secção corporal em estudo. ${ }^{5}$

Assiste-se, atualmente, à utilização crescente da PET/ CT para a pesquisa de metástases ósseas, recorrendo a vários radiofármacos, biomarcadores de diferentes comportamentos moleculares dessas metástases. São exemplos desses biomarcadores, a fluorodesoxiglicose-F18 (FDG-F18) que identifica atividade glicolítica aumentada, os mapeadores de características específicas de um tumor, como a colina-F18 (FCH-F18) que identifica a maior captação de colina pelas células tumorais do carcinoma da próstata e os análogos da somatostatina para os tumores neuroendócrinos ${ }^{6}$ e, ainda, o fluoreto de sódio-F18 (FNa-F18) que identifica o aumento da atividade osteoblástica. ${ }^{7}$

Com este trabalho pretendemos demonstrar a maior capacidade de deteção da PET/CT com FNa-F18 de alterações esqueléticas compatíveis com metástases ósseas em relação à $\mathrm{CO}$, à $\mathrm{PET} / \mathrm{CT}$ com FDG-F18 e à $\mathrm{PET} / \mathrm{CT}$ com $\mathrm{FCH}-\mathrm{F} 18$.

Procurámos, ainda, evidenciar que a superior capacidade de deteção da PET/CT com FNa-F18 leva a uma menor necessidade de realização de estudos adicionais para esclarecimentos complementares, quando comparada com a CO.

Foi, também, um objetivo deste trabalho comprovar a superior capacidade de deteção da componente molecular $\mathrm{PET}$ em relação à componente CT smart dose (CTsd) da técnica híbrida PET/CT com FNa-F18.

\section{MATERIAL E MÉTODOS \\ População estudada e metodologia}

Foram revistos os processos clínicos de 114 doentes, 65 mulheres e 49 homens, média de idades $62 \pm 10$ anos (16 - 82), que realizaram PET/CT com FNa-F18 para pesquisa de metástases ósseas, entre março de 2009 e março de 2016. Do total dos doentes incluídos no estudo, 58 (51\%) tinham carcinoma da mama, $41(36 \%)$ carcinoma da próstata, oito $(7 \%)$ carcinoma pulmonar e sete $(6 \%)$ outras condições clínicas (dois doentes com imagens suspeitas, em CT, de metástases ósseas de tumor de origem desconhecida, um com síndrome paraneoplásica, um com carcinoma do cólon, um com carcinoma da bexiga, um com carcinoma do seio piriforme e um com o diagnóstico de sarcoma).

Neste grupo de 114 doentes foi identificada a realização, num intervalo de tempo até seis meses, de outros exames de Medicina Nuclear também considerados com capacidade de deteção de metástases ósseas (CO, PET/ CT com FDG-F18 e PET/CT com FCH-F18) e indicados nas respetivas situações clínicas daqueles doentes. 
Garantimos que, nesse intervalo de tempo, não se registou nenhuma alteração significativa no quadro clínico/laboratorial dos doentes, nem ocorreu qualquer modificação na sua abordagem terapêutica. Os exames de PET/CT com FNa-F18 foram comparados com os resultados de cada uma daquelas modalidades imagiológicas. Foram registados os exames considerados positivos para pesquisa de lesões ósseas secundárias e foi comparada a percentagem de positividade. Foi também avaliado o desempenho das técnicas imagiológicas, através da identificação daquela que detetou maior número de lesões por doente.

Dos 114 doentes que realizaram PET/CT com FNa-F18, 49 também realizaram CO. A média ( $\pm \mathrm{DP})$, em dias, entre a realização da PET/CT com FNa-F18 e a CO foi de $68 \pm$ 62 (2 - 176). Neste subgrupo de 49 doentes, 32 (65,3\%) tinham carcinoma da mama, $10(20,5 \%)$ carcinoma da próstata, seis $(12,2 \%)$ carcinoma do pulmão e um $(2 \%)$ tinha imagens suspeitas, em CT, de metástases ósseas de tumor de origem desconhecida.

Dos 114 doentes que realizaram PET/CT com FNa-F18, 61 também realizaram PET/CT com FDG-F18. A média $( \pm$ $\mathrm{DP})$, em dias, entre a realização da PET/CT com FNa-F18 e a PET/CT com FDG-F18 foi de $35 \pm 50$ (1 - 181). Neste subgrupo de 61 doentes, $49(80,4 \%)$ tinham carcinoma da mama, seis (10\%) carcinoma do pulmão, um (1,6\%) carcinoma do cólon, um $(1,6 \%)$ imagens suspeitas, em CT, de metástases ósseas de tumor de origem desconhecida, um $(1,6 \%)$ tinha síndrome paraneoplásica, um $(1,6 \%)$ carcinoma do seio piriforme, um $(1,6 \%)$ carcinoma da bexiga e um $(1,6 \%)$ tinha o diagnóstico de sarcoma.

Dos 114 doentes que realizaram PET/CT com FNa-F18, 10 doentes, com carcinoma da próstata, também realizaram PET/CT com FCH-F18. A média ( $\pm D P$ ), em dias, entre a realização da PET/CT com FNa-F18 e a PET/CT com $\mathrm{FCH}-\mathrm{F} 18$ foi de $44 \pm 70$ (3 - 181).

Nos 114 doentes que realizaram PET/CT com FNa-F18, os achados da PET foram comparados com a CTsd e identificou-se qual a componente da técnica híbrida, molecular versus morfológica, que apresentou maior capacidade de deteção de alterações compatíveis com metástases ósseas.

Nos 49 doentes que realizaram CO e nos 114 doentes que realizaram $\mathrm{PET} / \mathrm{CT}$ com FNa-F18 registaram-se as situações em que foram sugeridos exames complementares adicionais para esclarecimento das alterações observadas.

\section{Protocolo de realização da PET/CT com FNa-F18}

Todos os doentes propostos para a realização da PET/ CT com FNa-F18 foram informados sobre os objetivos e procedimentos do exame tendo concordado com a sua realização e assinado um termo de consentimento informado.

O protocolo de aquisição do exame implicou a aquisição de imagens de todo o esqueleto 60 minutos após a administração endovenosa de $370 \mathrm{MBq}$ de FNa-F18. Os doentes foram posicionados em decúbito dorsal com os braços ao longo do corpo, num tomógrafo PET/CT GE Discovery ST. Os parâmetros de aquisição da CT para correção de ate- nuação e mapeamento anatómico foram os seguintes: 120 $\mathrm{kV}$, smart $m A$ : noise index de 35 com valores de corrente compreendidos entre 10 - $200 \mathrm{~mA}$, pitch 1,5:1, rotação de 0,5 s e uma espessura de corte de $3,75 \mathrm{~mm}$. O estudo de emissão PET foi obtido em modo 3D com um diâmetro de field of view (FOV) de $70 \mathrm{~cm}$ e com uma duração de três minutos de emissão por cada posição de mesa. Os dados foram recolhidos em list mode e reconstruídos usando um algoritmo de reconstrução iterativo 3D ordered subset expectation maximization (OSEM), com 20 subsets por duas iterações, matriz de 128 x 128 e um filtro pós-reconstrução de $5 \mathrm{~mm}$ full width at half maximum (FWHM).

\section{Interpretação das imagens da PET/CT com FNa-F18}

As imagens de PET/CT com FNa-F18 foram interpretadas por dois especialistas em Medicina Nuclear, por consenso, havendo conhecimento da história clínica do doente e acesso aos estudos analíticos e imagiológicos disponíveis.

Foi realizada análise semi-quantitativa calculando, para cada lesão, o valor de maximum standardized uptake value $\left(S U V_{\max }\right)$. A determinação do SUV max $_{\text {maseou-se na criação }}$ de um volume de interesse que envolvia completamente e apenas a lesão e serviu como indicador da intensidade de captação do radiofármaco pelas lesões detetadas.

O diagnóstico de metástases ósseas baseou-se na intensidade de captação de FNa-F18 e nas características tomodensitométricas na CT da PET/CT.

A captação de FNa-F18 foi considerada anómala e sugestiva da presença de metástases ósseas quando a sua intensidade foi superior à atividade registada no esqueleto normal. A caracterização estrutural e a localização anatómica destas alterações, facultada pelo componente CT da técnica, permitiu excluir patologia óssea benigna, como o compromisso articular inflamatório/degenerativo e outras situações benignas que também cursam com aumento da atividade osteoblástica.

\section{Análise estatística}

Para análise estatística dos dados foi usado o software SPSS (versão 23). Um $p$ inferior a 0,05 foi considerado significativo para todos os testes realizados. Os valores dos dados quantitativos são apresentados com média \pm desvio padrão (DP) e os qualitativos com n (\%). Para comparar a positividade entre exames foi usado o teste de McNemar. Para avaliação do desempenho foi usado o teste da Binomial. Para comparar, entre a PET/CT com FNa-F18 e a CO, a necessidade de exames para esclarecimentos complementares, foi usado o teste de Qui-quadrado.

\section{RESULTADOS}

Registou-se, em cada subgrupo de doentes, a frequência de exames considerados positivos para pesquisa de metástases ósseas e procedeu-se à avaliação comparativa dos resultados. Registou-se, ainda, a técnica que, para cada doente, revelou desempenho superior ao identificar maior número de lesões compatíveis com metástases ósseas. 
Tabela 1 - PET/CT com FNa-F18 versus Cintigrafia Óssea (49 doentes)

\begin{tabular}{lccccc}
\hline Técnica Imagiológica & $\begin{array}{c}\text { Positiva } \\
\mathbf{n}(\%)\end{array}$ & $\begin{array}{c}\text { Negativa } \\
\mathbf{n ~ ( \% )}\end{array}$ & $\begin{array}{c}\text { Duvidosa } \\
\mathbf{n}(\%)\end{array}$ & $\begin{array}{c}\text { Desempenho } \\
\text { Sobreponivel }\end{array}$ & $\begin{array}{c}\text { Desempenho } \\
\text { Superior }\end{array}$ \\
\hline PET/CT com FNa-F18 & $33(67 \%)$ & $16(33 \%)$ & $0(0 \%)$ & $25(51 \%)$ & $24(49 \%)$ \\
Cintigrafia ossea & $28(57 \%)$ & $16(33 \%)$ & $5(10 \%)$ & $25(51 \%)$ & $0(0 \%)$ \\
\hline
\end{tabular}

Tabela 2 - PET/CT com FNa-F18 versus PET/CT com FDG-F18 (61 doentes)

\begin{tabular}{lccccc}
\hline Técnica Imagiológica & $\begin{array}{c}\text { Positiva } \\
\mathbf{n}(\%)\end{array}$ & $\begin{array}{c}\text { Negativa } \\
\mathbf{n}(\%)\end{array}$ & $\begin{array}{c}\text { Duvidosa } \\
\mathbf{n}(\%)\end{array}$ & $\begin{array}{c}\text { Desempenho } \\
\text { Sobreponivel }\end{array}$ & $\begin{array}{c}\text { Desempenho } \\
\text { Superior }\end{array}$ \\
\hline PET/CT com FNa-F18 & $41(67 \%)$ & $20(33 \%)$ & $0(0 \%)$ & $25(41 \%)$ & $36(59 \%)$ \\
PET/CT com FDG-F18 & $27(44 \%)$ & $33(54 \%)$ & $1(2 \%)$ & $25(41 \%)$ & $0(0 \%)$ \\
\hline
\end{tabular}

Tabela 3 - PET/CT com FNa-F18 versus PET/CT com FCH-F18 (10 doentes)

\begin{tabular}{lccccc}
\hline Técnica Imagiológica & $\begin{array}{c}\text { Positiva } \\
\mathbf{n}(\%)\end{array}$ & $\begin{array}{c}\text { Negativa } \\
\mathbf{n}(\%)\end{array}$ & $\begin{array}{c}\text { Duvidosa } \\
\mathbf{n}(\%)\end{array}$ & $\begin{array}{c}\text { Desempenho } \\
\text { Sobreponível }\end{array}$ & $\begin{array}{c}\text { Desempenho } \\
\text { Superior }\end{array}$ \\
\hline PET/CT com FNa-F18 & $5(50 \%)$ & $5(50 \%)$ & $0(0 \%)$ & $6(60 \%)$ & $4(40 \%)$ \\
PET/CT com FCH-F18 & $2(20 \%)$ & $7(70 \%)$ & $1(10 \%)$ & $6(60 \%)$ & $0(0 \%)$ \\
\hline
\end{tabular}

\section{PET/CT com FNa-F18 versus cintigrafia óssea}

No sub-grupo de 49 doentes que realizaram PET/CT com FNa-F18 e CO, a PET/CT com FNa-F18 foi positiva para pesquisa de lesões compatíveis com metástases ósseas em 33 (67\%) doentes e negativa em 16 (33\%). A CO foi positiva para pesquisa de lesões compatíveis com metástases ósseas em 28 (57\%) doentes, negativa em 16 (33\%) e duvidosa em cinco (10\%). Embora a positividade de PET/CT com FNa-F18 seja maior que a de CO, a diferença encontrada não foi significativa $(p=0,063)$. A PET/CT com FNa-F18 apresentou um desempenho sobreponível à CO em 25 (51\%) dos doentes mas foi-lhe superior em 24 (49\%) em que detetou um maior número de lesões. Em comparação com a $\mathrm{CO}$, a proporção de doentes em que a $\mathrm{PET} / \mathrm{CT}$ com FNa-F18 detetou maior número de lesões compatíveis com metástases ósseas foi significativamente maior $(p<0,001)$. Estes resultados são apresentados na Tabela 1.

\section{PET/CT com FNa-F18 versus PET/CT com FDG-F18}

No subgrupo de 61 doentes que realizaram PET/CT com FNa-F18 e PET/CT com FDG-F18, a PET/CT com FNa-F18 foi positiva para pesquisa de lesões compatíveis com metástases ósseas em $41(67 \%)$ doentes e negativa em 20 (33\%). A PET/CT com FDG-F18 foi positiva para pesquisa de lesões compatíveis com metástases ósseas em 27 (44\%) doentes, negativa em 33 (54\%) e duvidosa em um (2\%) doente. A positividade apresentada pela PET/ CT com FNA-F18 foi significativamente maior do que a da PET/CT com FDG-F18 $(p<0,001)$.

A PET/CT com FNa-F18 apresentou um desempenho sobreponível à PET/CT com FDG-F18 em 25 (41\%) doentes tendo sido superior em 36 (59\%). A proporção de doentes em que a PET/CT com FNa-F18 detetou maior número de lesões compatíveis com metástases ósseas foi significativamente maior em comparação com a PET/CT com FDG-F18 $(p<0,001)$. Estes resultados são apresentados na Tabela 2.

\section{PET/CT com FNa-F18 versus PET/CT com FCH-F18}

No subgrupo de 10 doentes que realizaram PET/CT com FNa-F18 e PET/CT com FCH-F18, a PET/CT com FNa-F18 foi positiva para pesquisa de lesões compatíveis com metástases ósseas em cinco (50\%) doentes e negativa em cinco (50\%). A PET/CT com FCH-F18 foi positiva em dois $(20 \%)$ doentes, negativa em sete $(70 \%)$ e duvidosa em um (10\%). A positividade apresentada pela PET/CT com FNa-F18 foi maior que a conseguida com a PET/CT com $\mathrm{FCH}-\mathrm{F} 18$. A diferença não foi significativa $(p<0,250)$ mas trata-se de uma amostra de apenas 10 doentes.

A PET/CT com FNa-F18 apresentou um desempenho sobreponível à PET/CT com FCH-F18 em seis (60\%) doentes e foi superior em quatro $(40 \%)$ dos casos. A proporção de doentes em que a PET/CT com FNa-F18 detectou maior número de lesões compatíveis com metástases ósseas foi significativamente maior em comparação com a PET/CT com FCH-F18 $(p<0,001)$. Estes resultados são apresentados na Tabela 3.

\section{Componente molecular PET versus componente mor- fológica CT}

A componente molecular da PET/CT com FNa-F18 foi positiva para pesquisa de lesões compatíveis com metástases ósseas em 64/114 (56\%) doentes, negativa em 49/114 (43\%) e duvidosa em $1 / 114$ (1\%). A CTsd foi positiva para pesquisa de lesões compatíveis com metástases ósseas em 48/114 (42\%) doentes e negativa em 66/114 (58\%). A positividade apresentada pela PET/CT com FNa-F18 foi significativamente maior do que a positividade apresentada pela CTsd $(p<0,001)$.

A PET apresentou um desempenho sobreponível à CTsd em 74/114 (65\%) dos exames mas foi superior em 40/114 (35\%). A proporção de doentes em que a PET detectou maior número de lesões compatíveis com metástases ósseas foi significativamente maior em comparação com a CTsd $(p<0,001)$.

Verificou-se que, na população estudada, nenhuma das técnicas imagiológicas de comparação conseguiu ser 

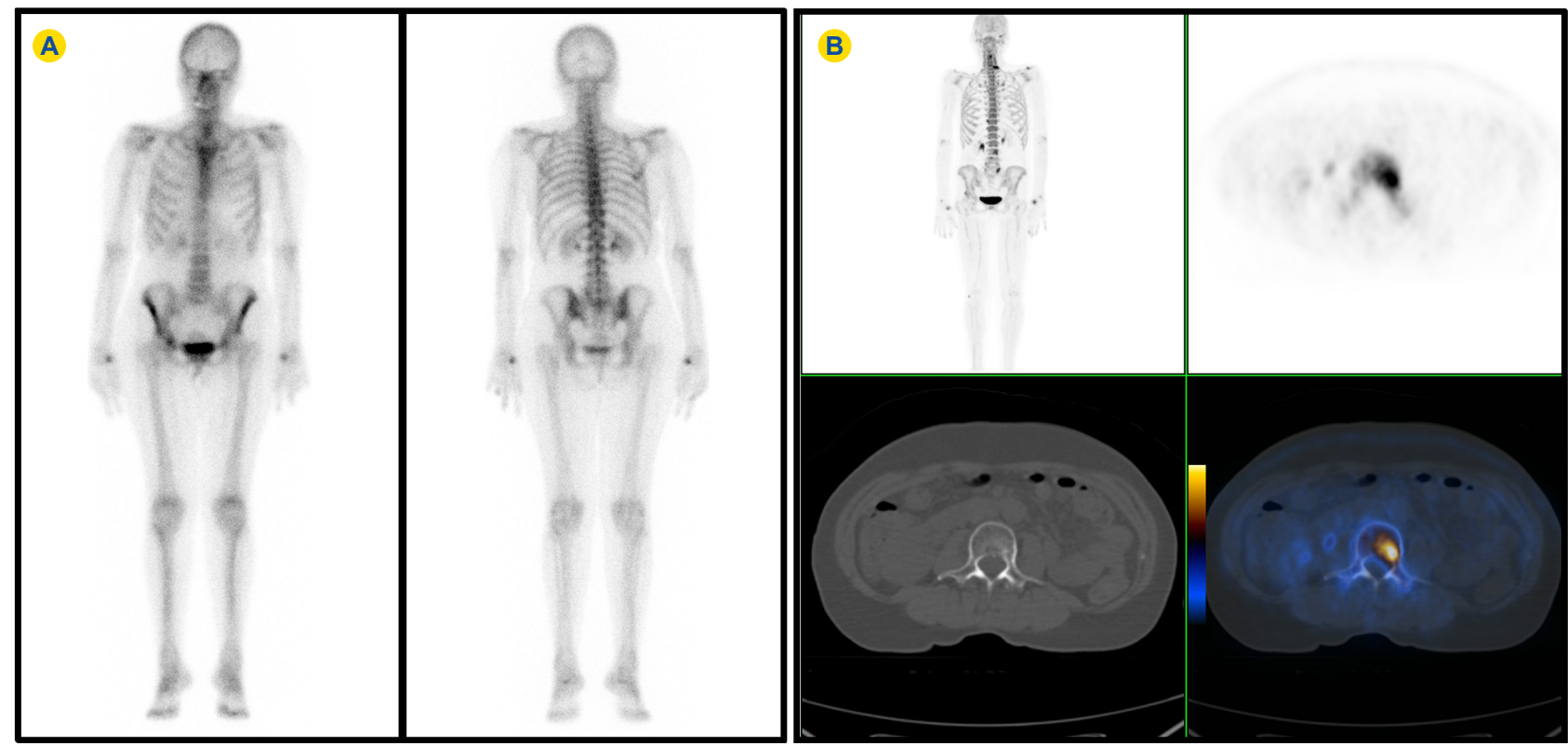

Figura 1 - Doente com carcinoma da mama tratado há cerca de 10 anos. Apresentava elevação do marcador tumoral e PET/CT com FDG-F18 sem evidência de doença. A cintigrafia óssea (A) não mostrou alterações mas a PET/CT com FNa-F18 (B) revelou alterações sugestivas de metastização óssea na coluna vertebral e na grelha costal, sem alterações no componente CT.
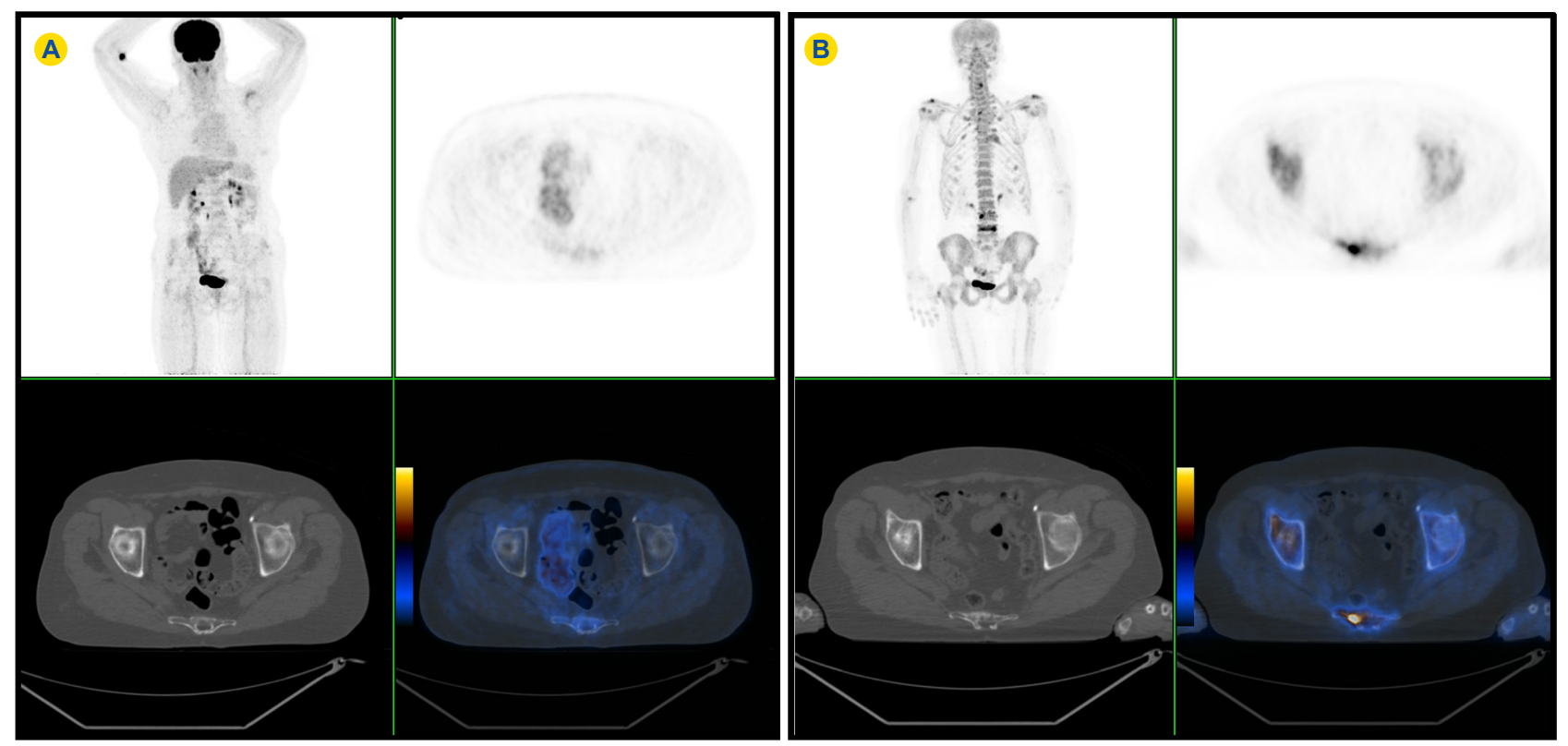

Figura 2 - Doente com carcinoma da mama tratado há cerca de 6 anos apresentando elevação do marcador tumoral. PET/CT com FDG-F18 sem alterações suspeitas (A) e PET/CT com FNa-F18 sugestiva de metastização óssea múltipla (B).

superior à $\mathrm{PET} / \mathrm{CT}$ com FNa-F18, quer em relação à positividade por exame, quer em relação ao número de lesões detetadas por doente.

Nas Fig.s 1, 2 e 3 apresentam-se exemplos de situações clínicas ilustrativas da superior capacidade de deteção da $\mathrm{PET} / \mathrm{CT}$ com FNa-F18, em relação à $\mathrm{CO}$ e à CTsd, à $\mathrm{PET} / \mathrm{CT}$ com FDG-F18 e à $\mathrm{PET/CT}$ com FCH-F18, respetivamente.

Da identificação dos doentes que necessitaram de outros exames complementares para melhor esclarecimento diagnóstico, após realização de PET/CT com FNa-F18 e após $\mathrm{CO}$, constatou-se que a necessidade de esclarecimento complementar foi referida em relação a 22/49 (45\%) dos doentes que realizaram CO e apenas em 4/114 (3,5\%) dos doentes que realizaram PET/CT com FNa-F18. Esta diferença foi significativa $(p<0,001)$.

\section{DISCUSSÃO}

O FNa-F18 é um radiofármaco PET disponível para estudo imagiológico do esqueleto. Foi usado pela primeira vez em 1962 mas, nessa altura, a sua utilização foi limitada pelas características técnicas dos equipamentos de deteção então disponíveis. Com o aparecimento dos equipamentos PET, o FNa-F18 foi reintroduzido na prática clínica encontrando-se em avaliação a sua utilização para a deteção de lesões ósseas. ${ }^{8} \mathrm{O}$ mecanismo de captação do 

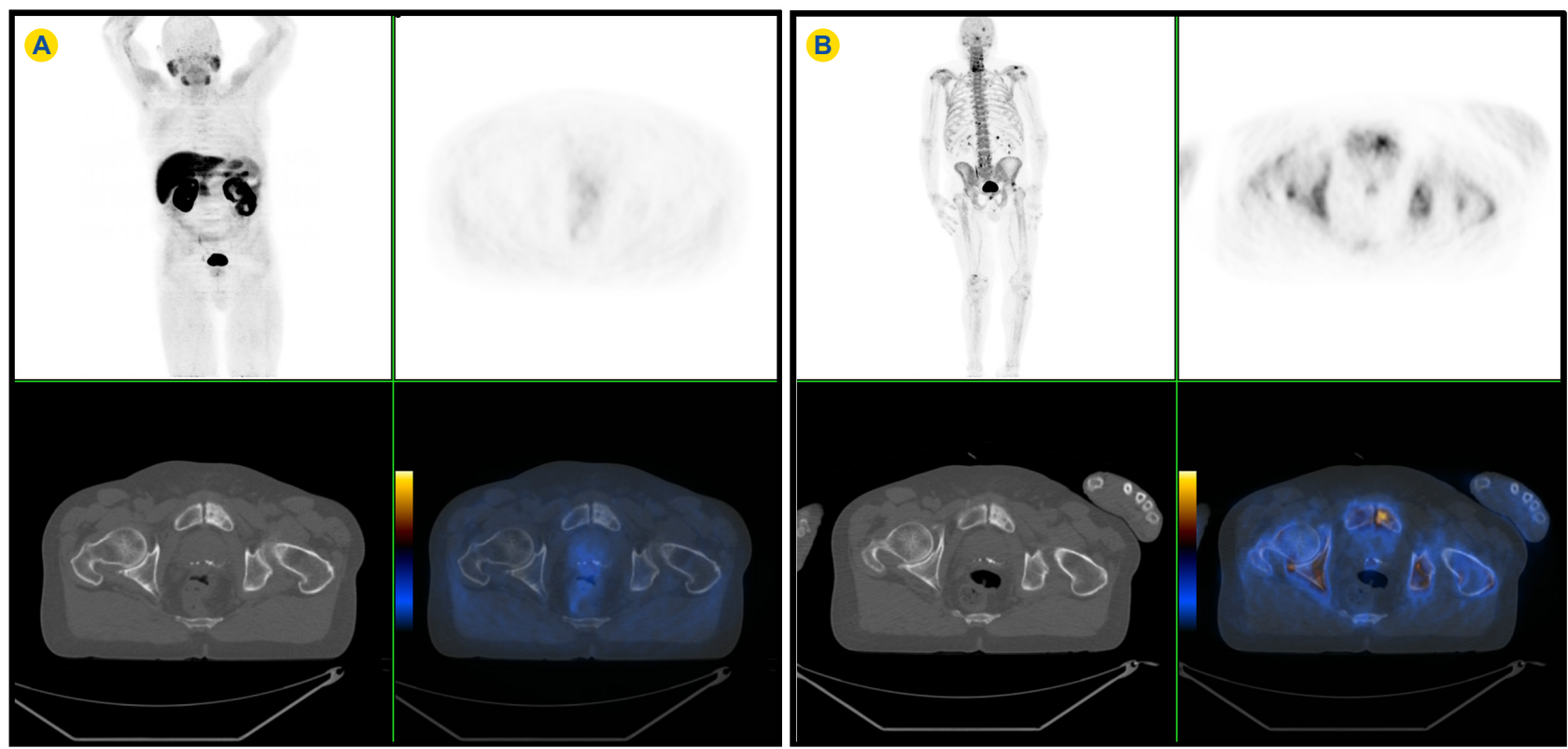

Figura 3 - Doente com carcinoma da próstata em recidiva bioquímica. PET/CT com FCH-F18 sem alterações suspeitas (A) e PET/CT com FNa-F18 sugestiva de metastização óssea na bacia e no crânio (B).

FNa-F18 é semelhante ao dos agentes utilizados na CO. Após a sua difusão dos capilares para o tecido ósseo, os iões fluoreto substituem os grupos hidroxilo dos cristais de hidroxiapatite formando fluoropatite que se deposita nos locais de turnover e remodelação óssea aumentados. ${ }^{9}$ As vantagens da $\mathrm{PET} / \mathrm{CT}$ com $\mathrm{FNa}-\mathrm{F} 18$ em relação à $\mathrm{CO}$ resultam das melhores características quer da técnica quer do radiofármaco. A PET/CT é tecnicamente vantajosa porque é uma modalidade imagiológica tomográfica e híbrida, com sensibilidade e resolução espacial superiores à $\mathrm{CO}$ planar, mesmo quando esta é complementada com imagens seccionais de SPET ou SPET/CT. O FNa-F18 tem características farmacocinéticas vantajosas porque praticamente não tem ligação às proteínas plasmáticas, ao contrário dos difosfonatos, sendo a sua clearance sanguínea mais rápida e praticamente completa. Além disso, a sua captação óssea é duas a três vezes superior. Estas características traduzem-se por imagens de melhor qualidade, com relações lesão/fundo e lesão/tecido ósseo normal, superiores. ${ }^{10}$ A dosimetria do $\mathrm{FNa}-\mathrm{F} 18$ e dos difosfonatos é semelhante, considerando-se que o acréscimo da dose absorvida devido à CT não é significativo. As novas tecnologias associadas à PET, como o time of flight (TOF) permitem administrar atividades bastante inferiores, cerca de metade das vulgarmente utilizadas, correspondendo a doses semelhantes, ou mesmo inferiores, às envolvidas na CO. ${ }^{11} \mathrm{O}$ tempo total de aquisição da PET/CT com FNa-F18 é inferior ao da $\mathrm{CO}$ resultando num exame mais cómodo de realizar. ${ }^{12}$ Estudos comparativos com a CO demonstram que a PET/ CT com FNa-F18 consegue detetar as metástases ósseas mais precocemente,,$^{13}$ apresenta sensibilidade e acuidade superiores e é mais fiável para excluir a existência de metástases ósseas. ${ }^{14-16}$ No nosso trabalho, limitados pela falta de um gold standard histológico para todas as lesões ósseas estudadas, não procurámos avaliar as característi- cas de sensibilidade, especificidade e acuidade diagnóstica das técnicas que comparámos. Admitimos, assim, implicitamente, a possibilidade de existência, para qualquer uma das técnicas em comparação, de um número indeterminado de falsos positivos e de falsos negativos. Sublinha-se, no entanto, que alguns estudos publicados atribuem à PET/ CT com FNa-F18 valores de sensibilidade e especificidade próximos dos $100 \%$ e consideram esta modalidade imagiológica como o exame de primeira linha para a pesquisa de metástases ósseas. ${ }^{16,17}$ Apresenta elevada especificidade porque a CT da PET/CT, ao caracterizar morfologicamente as alterações funcionais, contribui para a diferenciação entre lesões benignas e metástases ósseas. ${ }^{10}$ Permite, assim, otimizar o estadiamento, selecionar a melhor estratégia terapêutica na altura mais adequada ${ }^{18} \mathrm{e}$ é mais eficaz na avaliação da resposta ao tratamento instituído, ${ }^{19}$ tendo superior impacto na abordagem dos doentes oncológicos com metástases ósseas. ${ }^{20,21}$

$\mathrm{Na}$ nossa experiência, a PET/CT com FNa-F18 também apresentou maior capacidade de deteção que a $\mathrm{CO}$ (em $49 \%$ dos casos) e fê-lo de um modo mais assertivo, necessitando de exames complementares para melhor esclarecimento num número muito menor de doentes $(3,5 \%$ vs $45 \%$ ). A menor necessidade de realização de estudos adicionais para esclarecimentos complementares poderá ser, assim, uma outra vantagem da PET/CT com FNa-F18, a confirmar em estudos mais alargados e que incluam análise de custo-efetividade.

A PET/CT com FDG-F18 permite a deteção, simultaneamente, de metastização óssea e extra-óssea, o que pode constituir, por isso mesmo, uma vantagem no processo de avaliação global do doente oncológico. Atualmente é consensual que a PET/CT com FDG-F18 apresenta sensibilidade significativamente superior à $\mathrm{CO}$ para a deteção de metástases ósseas de natureza lítica e de metástases 
intra-medulares. ${ }^{22}$ Vários estudos que comparam a PET/CT com FDG-F18, a CT, a RM e a CO concluem que a PET/ CT com FDG-F18 e a RM de corpo inteiro têm acuidades semelhantes entre si e significativamente superiores às da CT e da CO ${ }^{2,23}$ Contudo, a PET/CT com FDG-F18, sendo sensível para a deteção de metástases osteolíticas, apresenta sensibilidade inferior para a deteção de metástases osteoblásticas $^{24}$ e tem, ainda, menor sensibilidade para a deteção das lesões a nível dos ossos do crânio devido à atividade fisiológica cerebral. ${ }^{25}$ Uma técnica imagiológica como a PET/CT com FNa-F18, capaz de identificar os dois tipos de metástases ósseas é, por isso, vantajosa. ${ }^{26}$ A PET/CT com FNa-F18 permite a identificação das metástases osteoblásticas $\mathrm{e}$, indiretamente, das metástases osteolíticas por identificar a reação osteoblástica que estas provocam no osso são adjacente, mesmo que essa reação seja mínima. Reconhece-se, contudo, que PET/CT com FDG-F18 tem impacto prognóstico superior ao identificar as metástases osteolíticas, de maior atividade metabólica e mais agressivas, associadas a piores prognósticos, com sobrevidas médias, global e livre de doença, inferiores. ${ }^{27}$ lagaru e colaboradores realizaram um estudo piloto, prospetivo, em que compararam a capacidade de avaliação da extensão da doença óssea com PET/CT com FNa-F18, com a CO, a PET/CT com FDG-F18 e, ainda, com a RM de corpo inteiro. Concluíram que a PET/CT com FNa-F18 foi superior a qualquer uma das outras técnicas. ${ }^{28}$ Numa meta-análise que comparou a acuidade diagnóstica da PET/CT com FNa-F18 com a CO e a PET/CT com FDG-F18, avaliando 20 artigos publicados, contendo um total de 1170 doentes, concluiu-se, também, que a PET/CT com FNa-F18 tem uma excelente capacidade diagnóstica para a deteção de metástases ósseas e revela-se vantajosa quando comparada com a CO e a PET/CT com FDG-F18. ${ }^{29}$ No nosso estudo comprovámos, também, a superior capacidade de deteção da $\mathrm{PET} / \mathrm{CT}$ com $\mathrm{FNa}-\mathrm{F} 18$ em relação à PET/CT com FDG-F18 (em 59\% dos casos).

No grupo de doentes com carcinoma da próstata, embora de pequena dimensão, a PET/CT com FNa-F18 também apresentou maior capacidade de deteção comparativamente à PET/CT com FCH-F18 (em 40\% dos casos). Os nossos resultados estão de acordo com outros trabalhos publicados que apontam no sentido da PET/CT com FCH-F18 apresentar valores de especificidade mais elevados para a deteção das metástases ósseas do carcinoma da próstata mas sensibilidade inferior à $\mathrm{PET/CT}$ com $\mathrm{FNa}$ -F18. ${ }^{30}$ No entanto, uma reconhecida vantagem da PET/CT com $\mathrm{FCH}-\mathrm{F} 18$ relativamente à $\mathrm{PET} / \mathrm{CT}$ com $\mathrm{FNa}-\mathrm{F} 18$ é a sua capacidade para detectar também metastização extra-óssea, nomeadamente a nível dos gânglios linfáticos.

$\mathrm{Na}$ nossa amostra de doentes demonstrámos, ainda, que num número elevado de doentes foi possível identificar, na componente PET, alterações sem tradução no componente CT (em 35\% dos casos), demonstrando a reconhecida maior precocidade de deteção da PET em relação à $\mathrm{CT}^{3}$

Este trabalho pretende ser um contributo para uma re- flexão sobre a melhor metodologia a adotar na pesquisa de metástases ósseas. As guidelines atuais continuam a incluir a CO como o método imagiológico a utilizar mas, cada vez mais, a literatura disponível enfatiza a superior capacidade de deteção da PET/CT com FNa-F18. ${ }^{20,31}$ Com a difusão dos equipamentos de PET/CT e a otimização da logística de distribuição do FNa-F18, é previsível que este exame venha a substituir a $\mathrm{CO}$, na prática clínica, não só nos doentes oncológicos ${ }^{18}$ como, também, nas patologias esqueléticas benignas. ${ }^{25,32}$

O trabalho que desenvolvemos apresenta várias limitações: a) trata-se de um estudo retrospetivo; b) o intervalo de tempo entre a realização das modalidades imagiológicas, embora num grande número dos casos seja aceitável, e tenha sido afastada a possibilidade de alterações significativas no quadro clínico, em alguns doentes é superior ao desejado; c) apesar dos achados imagiológicos terem sido valorizados de acordo com o contexto clínico, laboratorial e evolutivo de cada doente, não se procedeu à confirmação histológica das lesões compatíveis com metástases ósseas identificadas pela técnica - a confirmação histológica de todas as lesões identificadas não era nem viável nem ética. Admite-se, assim, a existência de um número indeterminado de falsos positivos e falsos negativos para qualquer uma das técnicas comparadas; d) a população estudada compreende um grupo heterogéneo de patologias as quais se podem traduzir por diversos tipos de lesões metastáticas ósseas (blásticas, líticas, mistas) apresentando diferentes graus de detetabilidade pelas várias técnicas que foram comparadas; e) o subgrupo de doentes em que se comparou a PET/CT com FNa-F18 e a PET/CT com FCH-F18 corresponde a um pequeno número de doentes; f) por último, deverá ser ressalvado que a CT foi realizada sem recurso à administração de contraste endovenoso, com parâmetros de aquisição (smart dose) otimizados para a PET/CT mas que não são os habitualmente usados na CT convencional.

\section{CONCLUSÃO}

Na população estudada, a PET/CT com FNa-F18 identificou um número elevado de alterações ainda sem tradução na componente CT da PET/CT com FNa-F18. Mostrou ser superior à $\mathrm{CO}$, com maior capacidade de deteção de lesões compatíveis com metástases ósseas e com menor necessidade de realização de outros estudos adicionais para esclarecimento complementar. Apresentou, ainda, uma superior capacidade de deteção em relação à $\mathrm{PET/CT}$ com FDG-F18 e à PET/CT com FCH-F18. Os resultados obtidos apontam no sentido de se vir a justificar a adoção da PET/CT com FNa-F18 como exame de primeira linha para a deteção de metastização óssea. No entanto, torna-se necessária a consolidação desta indicação através de mais estudos, prospetivos, desenhados por patologia, e que incluam análises de custo-efetividade.

\section{PROTEÇÃO DE PESSOAS E ANIMAIS}

Os autores declaram que os procedimentos seguidos estavam de acordo com os regulamentos estabelecidos 
pela Comissão de Ética e de acordo com a Declaração de Helsínquia da Associação Médica Mundial.

\section{CONFIDENCIALIDADE DOS DADOS}

Os autores declaram ter seguido os protocolos do seu centro de trabalho acerca da publicação dos dados de doentes.

\section{REFERÊNCIAS}

1. Even-Sapir E. Imaging of malignant bone involvement by morphologic, scintigraphic, and hybrid modalities. J Nucl Med. 2005;46:1356-67.

2. Yang HL, Liu T, Wang XM, Xu Y, Deng SM. Diagnosis of bone metastases: a meta-analysis comparing ${ }^{18}$ FDG PET, CT, MRI and bone scintigraphy. Eur Radiol. 2011;21:2604-17.

3. Rybak LD, Rosenthal DI. Radiological imaging for the diagnosis of bone metastases. Q J Nucl Med. 2001;45:53-64.

4. Schirrmeister H, Guhlmann A, Elsner K, Kotzerke J, Glatting G, Rentschler M, et al. Sensitivity in detecting osseous lesions depends on anatomic localization: planar bone scintigraphy versus $18 \mathrm{~F} \mathrm{PET}$. J Nucl Med. 1999;40:1623-9.

5. Kosuda S, Kaji T, Yokoyama H, Yokokawa T, Katayama M, Iriye T, et al. Does bone SPECT actually have lower sensitivity for detecting vertebral metastasis than MRI? J Nucl Med. 1996;37:975-8.

6. Chua S, Gnanasegaran G, Cook GJ. Miscellaneous cancers (lung, thyroid, renal cancer, myeloma, and neuroendocrine tumors): role of SPECT and PET in imaging bone metastases. Semin Nucl Med. 2009;39:416-30.

7. Ouvrier MJ, Vignot S, Thariat J. Place de l'imagerie nucléaire et de ses évolutions pour le diagnostic et le suivi des métastases osseuses. Bull Cancer. 2013;100:1115-24.

8. Blau M, Nagler W, Bender MA. Fluorine-18: a new isotope for bone scanning. J Nucl Med. 1962;3:332-4.

9. Cook GJ, Fogelman I. The role of positron emission tomography in the management of bone metastases. Cancer. 2000;88:2927-33.

10. Segall G, Delbeke D, Stabin MG, Even-Sapir E, Fair J, Sajdak R, et al. SNM practice guideline for sodium 18F-fluoride PET/CT bone scans 1.0. J Nucl Med. 2010;51:1813-20.

11. Ohnona J, Michaud L, Balogova S, Paycha F, Nataf V, Chauchat P, et al. Can we achieve a radionuclide radiation dose equal to or less than that of $99 \mathrm{mTc}$-hydroxymethane diphosphonate bone scintigraphy with a low-dose 18F-sodium fluoride time-of-flight PET of diagnostic quality? Nucl Med Commun. 2013:34:417-25.

12. Grant FD, Fahey FH, Packard AB, Davis RT, Alavi A, Treves ST. Skeletal PET with 18F-fluoride: applying new technology to an old tracer. J Nucl Med. 2008;49:68-78

13. Even-Sapir E, Mishani E, Flusser G, Metser U. 18F-Fluoride positron emission tomography and positron emission tomography/computed tomography. Semin Nucl Med. 2007;37:462-9.

14. Damle NA, Bal C, Bandopadhyaya GP, Kumar L, Kumar P, Malhotra $\mathrm{A}$, et al. The role of $18 \mathrm{~F}$-fluoride PET-CT in the detection of bone metastase in patients with breast, lung and prostate carcinoma: a comparison with FDG PET/CT and 99mTc-MDP bone scan. Jpn J Radiol. 2013;31:262-9.

15. Bastawrous S, Bhargava P, Behnia F, Djang DS, Haseley DR. Newer PET application with an old tracer: role of $18 \mathrm{~F}-\mathrm{NaF}$ skeletal PET/CT in oncologic practice. Radiographics. 2014;34:1295-316.

16. Li Y, Schiepers C, Lake R, Dadparvar S, Berenji GR. Clinical utility of (18)F-fluoride PET/CT in benign and malignant bone diseases. Bone. 2012;50:128-39.

17. Bortot DC, Amorim BJ, Oki GC, Gapski SB, Santos AO, Lima MC, et al. ${ }^{18} \mathrm{~F}-$ Fluoride PET/CT is highly effective for excluding bone metastases even in patients with equivocal bone scintigraphy. Eur J Nucl Med Mol

\section{CONFLITOS DE INTERESSE}

Os autores declaram não ter qualquer conflito de interesse relativamente ao presente artigo.

\section{FONTES DE FINANCIAMENTO}

Os autores declaram não ter recebido qualquer subsídio ou financiamento relativo ao presente artigo.

Imaging. 2012;39:1730-6.

18. Poulsen $\mathrm{MH}$, Petersen $\mathrm{H}$, Høilund-Carlsen PF, Jakobsen JS, Gerke O Karstoft J, et al. Spine metastases in prostate cancer: comparison of technetium-99m-MDP whole-body bone scintigraphy, [(18) F]choline positron emission tomography(PET)/computed tomography (CT) and [(18) F]NaF PET/CT. BJU Int. 2014;114:818-23.

19. Hughes CT, Nix JW. Role of sodium fluoride PET imaging for identification of bony metastases in prostate cancer patients. Curr Urol Rep. 2015;16:31.

20. Hillner BE, Siegel BA, Hanna L, Duan F, Quinn B, Shields AF. 18Ffluoride PET used for treatment monitoring of systemic cancer therapy: results from the National Oncologic PET Registry. J Nucl Med. 2015;56:222-8.

21. Even-Sapir E, Metser U, Mishani E, Lievshitz G, Lerman H, Leibovitch I. The detection of bone metastases in patients with high-risk prostate cancer: 99mTc-MDP Planar bone scintigraphy, single- and multi-field-ofview SPECT, 18F-fluoride PET, and 18F-fluoride PET/CT. J Nucl Med. 2006;47:287-97.

22. Fogelman I, Cook G, Israel O, Van der Wall H. Positron emission tomography and bone metastases. Semin Nucl Med. 2005;35:135-42.

23. Heusner T, Gölitz P, Hamami M, Eberhardt W, Esser S, Forsting M, et al. "One-stop-shop" staging: should we prefer FDG-PET/CT or MRI for the detection of bone metastases? Eur J Radiol. 2011;78:430-5.

24. Cook GJ, Houston S, Rubens R, Maisey MN, Fogelman I. Detection of bone metastases in breast cancer by 18FDG PET: differing metabolic activity in osteoblastic and osteolytic lesions. J Clin Oncol. 1998;16:33759.

25. Araz M, Aras G, Küçük Ö. The role of $18 \mathrm{~F}-\mathrm{NaF}$ PET/CT in metastatic bone disease. J Bone Oncol. 2015;4:92-7.

26. Yoon SH, Kim KS, Kang SY, Song HS, Jo KS, Choi BH, et al. Usefulness of (18)F-fluoride PET/CT in breast cancer patients with osteosclerotic bone metastases. Nucl Med Mol Imaging. 2013;47:27-35.

27. Piccardo A, Puntoni M, Morbelli S, Massollo M, Bongioanni F, Paparo F et al. 18F-FDG PET/CT is a prognostic biomarker in patients affected by bone metastases from breast cancer in comparison with 18F-NaF PET/ CT. Nuklearmedizin. 2015;54:163-72.

28. lagaru A, Young P, Mittra E, Dick DW, Herfkens R, Gambhir SS. Pilot prospective evaluation of 99mTc-MDP scintigraphy, 18F NaF PET/CT, 18F FDG PET/CT and whole-body MRI for detection of skeletal metastases. Clin Nucl Med. 2013;38:290-6.

29. Shen CT, Qiu ZL, Han TT, Luo QY. Performance of 18 F-fluoride PET or PET/CT for the detection of bone metastases: a meta-analysis. Clin Nucl Med. 2015;40:103-10

30. Vali R, Loidl W, Pirich C, Langesteger W, Beheshti M. Imaging of prostate cancer with PET/CT using (18)F-Fluorocholine. Am J Nucl Med Mol Imaging. 2015;5:96-108.

31. Wondergem M, van der Zant FM, van der Ploeg T, Knol RJ. A literature review of $18 \mathrm{~F}$-fluoride PET/CT and $18 \mathrm{~F}$-choline or $11 \mathrm{C}$-choline PET/CT for detection of bone metastases in patients with prostate cancer. Nucl Med Commun. 2013;34:935-45.

32. Even-Sapir E. ${ }^{18} \mathrm{~F}$-fluoride PET/computed tomography imaging. PET Clin. 2014;9:277-85 